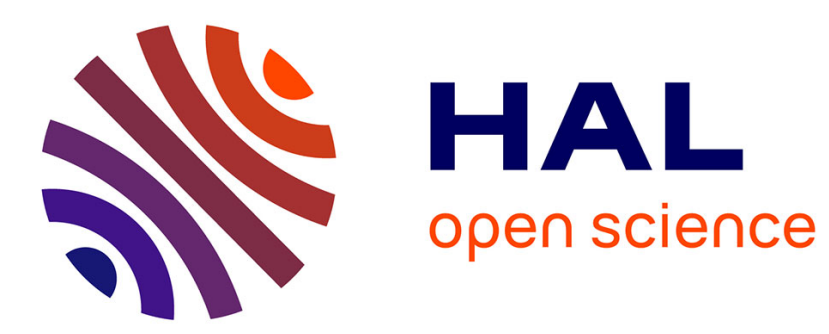

\title{
Faim et décisions intertemporelles : littérature expérimentale et illustration empirique
}

Youenn Loheac

\section{To cite this version:}

Youenn Loheac. Faim et décisions intertemporelles: littérature expérimentale et illustration empirique. Revue Economique, Presses de Sciences Po, 2019, 7, pp.368-381. halshs-02472148

\section{HAL Id: halshs-02472148 https://halshs.archives-ouvertes.fr/halshs-02472148}

Submitted on 10 Feb 2020

HAL is a multi-disciplinary open access archive for the deposit and dissemination of scientific research documents, whether they are published or not. The documents may come from teaching and research institutions in France or abroad, or from public or private research centers.
L'archive ouverte pluridisciplinaire HAL, est destinée au dépôt et à la diffusion de documents scientifiques de niveau recherche, publiés ou non, émanant des établissements d'enseignement et de recherche français ou étrangers, des laboratoires publics ou privés. 


\title{
Faim et décisions intertemporelles : littérature expérimentale et illustration empirique
}

\author{
Youenn LOHEAC ${ }^{1}$
}

\section{Résumé}

La faim semble influencer les préférences intertemporelles. Cet état physiologique est associé à une augmentation des taux d'escomptes, d'une part, et à une préférence pour les aliments « moins sains », d'autre part. La problématique d'arbitrage intertemporel en relation avec l'état de satiété est explorée à travers plusieurs expériences issues de la littérature et illustrée par une étude empirique mettant en relation des moments de la journée associés aux repas (et par extension à la faim) et des préférences intertemporelles. Les résultats montrent une hausse de la préférence pour le présent associée à la faim. Ils sont analysés à travers les notions d'escompte et de biais de projection.

\section{Hunger and intertemporal decisions: experimental literature and empirical illustration}

\section{Abstract}

Hunger seems to influence intertemporal preferences. This physiological state is associated with an increase in discount rates, on the one hand, and a preference for "less healthy" foods, on the other hand. The problem of intertemporal trade-off in relation with the state of satiety is explored through several experiments from the literature and illustrated by an empirical study between moments of the day associated with meals (and hence hunger) and intertemporal preferences. The results show an increase of preference for present linked to hunger. Results are analyzed with discount rates and projection bias.

Mots clés : décisions intertemporelles, consommation, expériences, satiété.

Keywords: intertemporal decisions, consumption, experiment, satiety.

Classification JEL : C91, D12, D15, D91

\footnotetext{
${ }^{1}$ - Brest Business School et CREM-CNRS, Université Rennes 1. Correspondance : Brest Business School - 2 avenue de Provence - 29200 Brest, France. Email : youenn.loheac@brest-bs.com

Remerciements : L'auteur remercie le groupe des lauréats 2016 de la Fondation pour les Sciences Sociales pour les échanges enrichissants, ainsi que François Dubet qui nous a accompagné en tant que Directeur Scientifique. Il remercie également Angela Sutan qui lui a permis de recueillir les données utilisées. Il remercie Xavier Gassmann et Mélody Leplat pour leur soutien sur le fond et la forme. Enfin, il remercie grandement le rapporteur anonyme pour ses commentaires.

Ce travail a été effectué avec le soutien de la Fondation pour les Sciences Sociales, sous l'égide de la Fondation de France.
} 


\section{INTRODUCTION}

Selon la théorie de l'utilité escomptée, les décisions intertemporelles sont réputées stables. Seule une modification du taux d'escompte peut les modifier. Cependant, cela est en partie rejeté par l'observation des comportements individuels (Frederick et al. [2002]). En effet, les choix apparaissent influencés par des modifications de l'environnement de décision ou de l'état du décideur lui-même. Le modèle de l'escompte hyperbolique ou quasihyperbolique (Laibson [1997]) permet d'expliquer qu'un individu orienté vers le futur choisit l'option la plus proche dans le temps en raison de son caractère immédiat. Selon les modèles relatifs aux signaux (Laibson [2001]; Bernheim et Rangel [2004]), les préférences individuelles liées aux perceptions temporelles peuvent être modifiées par un événement ponctuel ayant lui-même des conséquences sur les décisions de consommation. Enfin, Loewenstein [1996], [2000] propose un cadre où les arbitrages intertemporels peuvent être modifiés par l'état émotionnel ou physiologique (facteurs viscéraux) dans lequel est l'individu. Il postule que la compréhension des facteurs viscéraux vécus au moment de la décision de consommation est nécessaire pour appréhender les arbitrages intertemporels. Parmi ces facteurs, la faim est identifiée comme ayant un effet en termes de «perte de contrôle», situation au cours de laquelle un individu agit contre son propre intérêt, notamment futur.

La faim (et inversement la satiété) intervient dans la commande des prises alimentaires (Bellisle [2005]). Celles-ci sont aussi dépendantes de l'environnement du consommateur (Schwartz et al. [2017]) et de processus cognitifs (Cornil [2017]). Les prises alimentaires, discontinues, assurent les besoins énergétiques continus nécessaire au maintien et à l'activation des fonction vitales du corps humain. Le système physiologique associé renvoie à l'axe tractus gastro-intestinal cerveau et implique deux types de signaux (mécaniques et endocrines) mettant en action un ensemble de phénomènes associant circulation, tissus, organes et système nerveux (Pénicaud et Fioramonti [2015]). Trois hormones sont mises en avant dans l'alternance entre faim et satiété : ghréline, insuline et leptine (Bellisle [2005]; Niwano et al. [2009] ; Heisler et Lam [2017], par exemple). Schématiquement, une baisse de la leptine, de la glycémie (insuline) et une hausse de la ghréline déclenchent la sensation de faim et l'ingestion d'aliments. Celle-ci active un ensemble de mécanismes régulant le traitement et l'assimilation des nutriments, régulant la quantité d'aliments ingérés (et/ou la durée du repas), et conduisant à la satiété. Si la glycémie n'est pas le seul facteur de la faim, 
elle est souvent mise en avant dans sa relation à d'autres comportements. En effet, selon la revue de littérature d'Orquin et Kurzban [2016], elle pourrait venir perturber la capacité d'auto-contrôle d'un individu et ainsi augmenter son impatience. Ils soulignent qu'une faible glycémie augmente le taux d'escompte (préférence pour le présent) pour la nourriture, et dans une moindre mesure pour l'argent ou d'autres biens.

La préférence pour le présent est un attribut de l'arbitrage intertemporel, lorsqu'un individu est confronté à un choix entre une option proche dans le temps et une option plus éloignée. Le choix de l'option la plus proche dans le temps peut être justifiée par deux comportements. Selon la théorie de l'utilité escomptée, un individu ayant un fort taux d'escompte (impatient) choisira toujours cette option, toute chose égale par ailleurs (Frederick et al. [2002]). Les modèles de l'escompte hyperbolique ou quasi-hyperbolique (Laibson [1997]) admettent qu'un individu soit patient (faible taux d'escompte) lorsque les deux options (ayant la même durée entre elles) sont assez éloignées dans le temps et qu'il soit plus impatient lorsqu'elles sont plus proches. Ce phénomène est appelé inversion des préférences. La préférence pour le présent est alors stimulée par la satisfaction associée au gain immédiat, et pourrait être accentuée par la faim. Dans une forme dynamique, la préférence pour le présent peut apparaître lorsqu'un arbitrage porte sur le futur et qu'il est modifié lorsque le futur devient présent (incohérence temporelle dynamique; Halevy [2015)). Cette incohérence temporelle est interprétée à travers le biais de de projection (Loewenstein et al. [2003]). Selon ce principe, lorsque les individus anticipent leurs décisions futures, ils ont tendance à sousestimer la proximité pouvant exister entre leurs préférences présentes et leurs préférences futures, sans intervention d'un taux d'escompte. Ils peuvent ainsi penser que s'ils ne résistent pas à un aliment aujourd'hui, ils seront tout à fait capables de le faire plus tard. Loewenstein [1996], [2000], [2005] aborde également cette question en termes de distance empathique, c'est-à-dire la capacité à se mettre à la place de soi-même dans le futur ou dans un environnement de décision différent de celui connu actuellement. Une distorsion de cette empathie est assimilée au biais de projection. Les facteurs viscéraux tels que la faim sont alors associés à ces phénomènes.

Les arbitrages en faveurs du présent, que ce soit en raison de l'impatience ou du biais de projection, peuvent-il être renforcés par l'état de faim? Les relations précédemment identifiées permettent de poser les hypothèses sous-jacentes suivantes.

H1. La faim augmente le taux d'escompte

H1bis. La faible glycémie augmente la faim et l'impatience

H2. La faim présente a un effet sur les préférences futures (biais de projection) 
H3. La faim modifie les préférences anticipées lorsque celles-ci deviennent présentes (biais de projection et cohérence temporelle dynamique)

Ces hypothèses sont abordées dans la prochaine section à travers la littérature expérimentale. Puis, dans la section suivante, nous illustrons ce propos à travers une analyse empirique mettant en relation des décisions intertemporelles avec les repas. Nous concluons sur les pistes de recherches et d'interventions.

\section{LES EFFETS DE LA FAIM SUR LES ARBITRAGES INTERTEMPORELS : APPROCHES EXPERIMENTALES}

Les expériences mobilisées sont décrites dans le tableau 1 (incitations, mesures, horizons temporels, nombre de participants). Elles ne fournissent pas d'autre définition de la faim que celle renvoyant aux facteurs viscéraux et à leurs effets en termes d'états « chaud »et «froid» (Loewenstein [1996], [2000], [2005]). L'identification de cet état n'est pas homogène : autodéclaration, mesure de glycémie, échelle de satiété, et/ou contrôle par l'heure de l'expérience (avant ou après un repas) ou par l'ingestion d'aliments

Tableau 1. Description des expériences mobilisées relatives à la relation entre faim et arbitrages intertemporels

\begin{tabular}{|c|c|c|c|c|}
\hline Auteurs & Incitations & Expérience & $\begin{array}{l}\text { Horizon } \\
\text { temporel }\end{array}$ & Participants \\
\hline $\begin{array}{l}\text { Ashton } \\
\text { [2015] }\end{array}$ & oui & $\begin{array}{l}\text { Taux d'escompte } \\
\text { Argent } \\
\text { Contrôle du repas } \\
\text { Faim déclarée }\end{array}$ & 7 à 35 jours & 143 étudiants \\
\hline $\begin{array}{l}\text { Briz et al. } \\
{[2015]}\end{array}$ & oui & $\begin{array}{l}\text { Consentement à payer } \\
\text { Sandwich } \\
\text { Contrôle du repas }\end{array}$ & Une semaine & 252 étudiants \\
\hline $\begin{array}{l}\text { De-Magistrie } \\
\text { et Gracia } \\
{[2016]}\end{array}$ & oui & $\begin{array}{l}\text { Consentement à payer } \\
\text { Portion de fromage } \\
\text { Contrôle du repas } \\
\text { Faim déclarée }\end{array}$ & Un jour & 98 consommateurs \\
\hline $\begin{array}{l}\text { Gilbert et al. } \\
\text { [2002] }\end{array}$ & non & $\begin{array}{l}\text { Liste / achats } \\
\text { Aliments } \\
\text { Ingestion muffin }\end{array}$ & Non défini & 135 acheteurs \\
\hline $\begin{array}{l}\text { Kuhn et al. } \\
\text { [2017] }\end{array}$ & oui & $\begin{array}{l}\text { Taux d'escompte } \\
\text { Argent } \\
\text { Ingestion boisson } \\
\text { (sucre ou éduclcorant) }\end{array}$ & $\begin{array}{l}5,10,15 \\
\text { semaines }\end{array}$ & 280 étudiants \\
\hline Read et van & oui & Choix & Une semaine & 200 salariés \\
\hline
\end{tabular}




\begin{tabular}{|l|l|l|l|l|}
\hline $\begin{array}{l}\text { Leeuwen } \\
{[1998]}\end{array}$ & & $\begin{array}{l}\text { Fruit / barre } \\
\text { chocolatée } \\
\text { Contrôle du repas }\end{array}$ & & \\
\hline $\begin{array}{l}\text { Reuben et al. } \\
{[2010]}\end{array}$ & oui & $\begin{array}{l}\text { Taux d'escompte } \\
\text { Argent, chocolat } \\
\text { Faim déclarée }\end{array}$ & Une semaine & 57 étudiants \\
\hline $\begin{array}{l}\text { Skrynka et } \\
\text { Vincent } \\
{[2017]}\end{array}$ & non & $\begin{array}{l}\text { Taux d'escompte } \\
\text { Argent, Chocolat, } \\
\text { musique } \\
\text { Contrôle du repas } \\
\text { Mesure glycémie } \\
\text { Faim déclarée }\end{array}$ & $\begin{array}{l}\text { De } 1 \text { heure à } \\
1 \text { an }\end{array}$ & 50 étudiants \\
\hline $\begin{array}{l}\text { Wang et } \\
\text { Huangfu } \\
{[2017]}\end{array}$ & non & $\begin{array}{l}\text { Taux d'escompte } \\
\text { Argent } \\
\text { Ingestion glucose } \\
\text { Faim déclarée }\end{array}$ & $\begin{array}{l}\text { De 1 à 1112 } \\
\text { jours }\end{array}$ & 147 étudiants \\
\hline
\end{tabular}

\section{H1. La faim augmente le taux d'escompte}

Dans l'étude de la relation entre le taux d'escompte et la faim, sans hypothèses relatives à un état futur de l'individu, le biais de projection n'est pas testé. Reuben et al. [2010] mettent en relation la faim avec des taux d'escomptes mesurés pour de l'argent et du chocolat. Ils constatent que le taux d'escompte est plus élevé pour le chocolat que pour l'argent (sujets plus impatients avec le chocolat). Ils observent en complément que le niveau d'impatience est fortement corrélé entre les deux taux d'escomptes, notamment pour les sujets qui aiment le chocolat et qui ont faim. Ils établissent ainsi une relation positive entre la faim et l'impatience. Deux autres expériences intègrent une plus grande maitrise de l'état physiologique des participants. Ashton [2015] met en relation plusieurs états physiologiques (faim, fatigue cognitive) avec des arbitrages intertemporels monétaires. Ses résultats montrent que la faim augmente l'impatience, et plus particulièrement celle associée à des gains immédiats, révélant un escompte hyperbolique. Par ailleurs, Skrynka et Vincent [2017] mettent en relation la faim avec les arbitrages intertemporels dans trois domaines (argent, barres de chocolat, morceaux de musique à télécharger). Le taux d'escompte pour les barres de chocolat est significativement plus élevé en état de faim, mais il n'y a aucune différence entre les deux états pour la musique et l'argent. Par ailleurs, si la faim subjective est un bon prédicteur du taux d'escompte (relation positive), la relation avec la glycémie n'est pas significative. En conclusion, dans le cadre de choix intertemporels, la faim augmente le taux d'escompte (pour de l'argent et/ou un aliment - ici du chocolat) et a fortiori positionne les individus dans un arbitrage en faveur du présent ou du plus court terme. 
H1bis. La faible glycémie augmente la faim et l'impatience

Dans leur expérience, Wang et Huangfu [2017] montrent que l'ingestion de glucose réduit le taux d'escompte, c'est-à-dire qu'elle rend les options futures plus attractives, et que ce phénomène est lié à la sensation de faim ressentie. Par ailleurs, Kuhn et al. [2017] observent que l'ingestion d'une boisson réduit significativement la préférence pour le présent, sans différence entre la boisson sucrée et celle avec édulcorants. Ainsi, la faim, potentiellement associée à une faible glycémie, semble avoir un effet sur la préférence pour le présent. Cependant, la réduction de la faim (diminuant la préférence pour le présent) peut être obtenue par un aliment sans calories mais remplissant l'estomac. Il apparaît ainsi que d'autre paramètres physiologiques de la faim soient à prendre en compte.

\section{H2. La faim présente a un effet sur les préférences futures (biais de projection)}

Deux expériences abordent cette hypothèse en distinguant le biais de projection de l'impulsivité, mais n'impliquent pas de mesure de taux d'escomptes. De-Magistrie et Gracia [2016] observent les effets de la faim présente et future sur l'évaluation d'un aliment futur (consentement à payer -CAP- pour une portion de fromage le jour suivant). Les traitements déterminent l'état des participants le jour même et le lendemain : (1) faim-faim, (2) satiétéfaim, (3) faim-satiété, et (4) satiété-satiété. Le protocole permet de tester le biais de projection dans la mesure où le produit futur est évalué en fonction des états présents et futurs. Le premier résultat est que la faim future augmente le CAP, quel que soit l'état de satiété présent (traitements 1 et 2). La faim future génère ainsi une impulsion présente à payer plus cher le produit futur (pas de biais de projection; il serait apparu si le CAP avait été plus faible dans le traitement 2). Le traitement 3 implique quant à lui un biais de projection. En effet, même si l'état futur est la satiété, la faim présente augmente le CAP. Il y a dans ce cas une influence de la faim présente sur la décision future qui n'apparait pas lorsque les états présent et futur sont la satiété (traitement 4). En conclusion, la faim présente induit une hausse du CAP pour le produit futur alors même que l'individu sait qu'il sera à ce moment en état de satiété. L'expérience conduite par Briz et al. [2015] est sensiblement différente. Elle mesure l'effet de la faim présente sur l'évaluation d'un aliment présent et d'un aliment futur. Elle ne considère pas la faim future, c'est l'aliment qui est « déplacé » dans le temps. Le résultat principal est 
que deux effets antagonistes apparaissent dans les CAP : la distance temporelle réduit la valeur attribuée à l'aliment, mais la faim présente l'augmente. Le premier effet correspond à la préférence pour le présent, l'attente est compensée par une baisse de la valeur. Le deuxième effet peut être associé à une impulsivité. Les auteurs identifient un troisième effet qui serait le cumul des deux précédents. Ils l'attribuent au biais de projection, c'est-à-dire que ne connaissant pas leur état futur, les sujets vont se baser sur leur état présent pour estimer leur état futur.

Si ces expériences mettent en avant un phénomène de biais de projection dans lequel la faim présente augmente la valeur attribuée à un aliment futur, leurs résultats connaissent une limite et une contradiction. La limite est liée au fait que les mêmes individus ne sont pas exposés aux différents états, ce qui ne permet pas d'envisager des mesures intra-individuelles correspondant aux évaluations d'arbitrages intertemporels. Nous sommes proches de protocoles relevant de l'étude de la stationnarité (Halevy [2015]), mais sans dimension individuelle. La contradiction quant à elle est associée à l'hypothèse 1, non rejeté précédemment, selon laquelle la faim augment le taux d'escompte. Selon cette hypothèse, le CAP pour un bien futur devrait être plus faible en état de faim qu'en état de satiété. C'est l'inverse qui est observé dans ces expériences. En effet, la faim se traduit par une hausse de la valeur du produit futur. Le biais de projection pourrait intervenir ici en contrecarrant l'effet de préférence pour le présent, mais cela n'est pas testé. Pour cela, il faudrait observer la décision relative au moment où le futur devient présent.

\section{H3. La faim modifie les préférences anticipées lorsque celles-ci deviennent présentes (biais de projection et cohérence temporelle dynamique)}

Cette dernière hypothèse aborde la question de la cohérence temporelle dynamique à travers l'observation de l'effet de la faim sur la modification d'une anticipation lorsque celleci devient réalité (lorsque le futur devient présent). Contrairement aux expériences précédentes portant sur des évaluations monétaires, celles-ci portent sur des choix observés de produits. Le fait stylisé associé est l'idée selon laquelle nous achetons des aliments «moins sains » lorsque nous avons faim. Dans une expérience conduite en supermarché, Gilbert et al. [2002] observent que les participants en état de faim ont moins respecté leur liste de course que ceux qui étaient en état de satiété. De plus, ils ont dépensé significativement plus d'argent sur la partie hors liste. La faim les a fait dévier de leurs prévisions, sans que les incitations 
n'aient changé. Cela correspondrait à un biais de projection. L'expérience de Read et van Leeuwen [1998] introduit la possibilité de modifier la décision lorsque le futur devient présent, sachant qu'au moment de la première décision les états présent et futur sont connus. Elle permet ainsi une distinction entre l'effet de l'escompte et le biais de projection. Les participants font un choix présent relatif à des options futures (un fruit ou une barre chocolatée dans une semaine). Lorsque futur devient présent (la semaine suivante), ils choisissent à nouveau entre les deux produits, sans que ne choix initial ne soit rappelé. Les participants sont répartis entre quatre traitements induisant les états présent et futur : (1) faimfaim, (2) satiété-faim, (3) faim-satiété, et (4) satiété-satiété. Un premier résultat valide l'hypothèse d'empathie temporelle (capacité à se mettre dans la peau du son soi futur) en relation avec la faim future. En effet, quel que soit leur état initial, les participants des traitements avec une faim future (1 et 2) choisissent plus fréquemment (écart d'un tiers) les barres chocolatées dans le futur, que ceux qui sont positionnés dans une satiété future (traitements 3 et 4). Cependant, un deuxième résultat montre que, quel que soit le traitement, un nombre significatif de participants ayant préféré un fruit dans le futur modifient leur choix pour une barre chocolaté lorsque le futur devient présent, au point que les proportions de choix pour ces barres dans les différents traitements sont comparables. Selon les auteurs, il ne s'agit pas ici de biais de projection mais d'un phénomène d'incohérence temporelle soutenue par l'escompte hyperbolique, dans la mesure où les statuts de faim ou de satiété ne jouent pas. Dans ce cadre, le biais de projection consisterait à ignorer la préférence pour le présent dans le futur.

En conclusion, à partir des expériences décrites, nous pouvons établir trois résultats et quelques modérations. Premièrement, la faim semble augmenter le taux d'escompte pour certains biens (argent, aliments) sans que cela ne soit systématique. Si le mécanisme physiologique sous-jacent mis en avant est la variation du taux de glycémie, celle-ci n'est pas le seul paramètre entrant en jeu dans le cycle faim-satiété. Des études associant les autres mécanismes hormonaux pourraient être nécessaires. Deuxièmement, la faim présente semble augmenter les CAP pour un aliment futur, quelque soit l'état physiologique futur. Le phénomène de biais de projection est ici mis en avant par les auteurs. Troisièmement, la faim modifie les décisions anticipées, lorsque le futur devient présent. Si le biais de projection peut être avancé comme explication dans ce cadre, celle de l'incohérence temporelle dynamique est également pertinente. Lorsque le futur devient présent, la faim implique une plus forte préférence pour le présent et modifie donc l'anticipation qui avait été faite. Ces éléments 
nécessitent des explorations complémentaires. Dans la section suivante, nous revenons sur l'hypothèse 1 relative à la relation entre la faim et l'escompte.

\section{ILLUSTRATION EMPIRIQUE : LES ARBITRAGES INTERTEMPORELS AU COURS DE LA JOURNEE}

Nous testons dans cette illustration la relation entre la faim et les arbitrages intertemporels pour deux objets (argent et chocolat) en nous appuyant sur le cycle, particulièrement stable, des repas au cours de la journée, en France. En effet, selon De Saint Pol et Ricroch [2012] et Lhuissier et al. [2013], les français prennent trois repas par jours à des horaires relativement fixes : le petit-déjeuner (de $6 \mathrm{~h}$ à $10 \mathrm{~h}$ ), le déjeuner (de $12 \mathrm{~h}$ à $14 \mathrm{~h}$ ) et le diner (de 19h à 21h). L'hypothèse posée est que la faim entraine une hausse de l'escompte avant ou au moment du repas.

Les données sont issues d'une expérience en ligne portant sur des choix dans le risque et dans le temps afin de comparer les décisions individuelles en fonction de l'incitation (argent versus chocolat), à la façon de Reuben et al. [2010]. Elle présente l'intérêt de pouvoir identifier l'heure à laquelle le participant a répondu à l'enquête et ainsi de le positionner dans le cycle des repas au cours de la journée. Les données ont été recueillies en novembre 2015 et en novembre 2016 auprès de 451 étudiants de Licence 3 d'un même établissement. L'expérience contenait des questions hypothétiques et des questions avec des incitations réelles (les seules utilisées ici). Pour ces dernières, un nombre connu de répondants étaient tiré au sort afin de déterminer les gains individuels.

Les données utilisées portent sur les décisions intertemporelles relatives à deux types de récompenses : le chocolat l'argent. Les participants ont pris 20 décisions (10 pour chaque récompense) portant sur des enjeux relativement faibles. Pour chacune, ils faisaient un choix entre un gain dans une semaine $\left(1,35 € / 9\right.$ mignonettes de chocolat $\left.{ }^{2}\right)$ et un gain plus élevé dans neuf semaines (qui prend les valeurs successives suivantes : 1,50€;1,65€;1,80€; $1,95 € ; 2,10 € ; 2,25 € ; 2,40 € ; 2,55 € ; 2,70 € ; 2,85 € / 10 ; 11 ; 12 ; 13 ; 14 ; 15 ; 16 ; 17 ; 18$; 19 mignonettes). Chaque série de décision permet de calculer un score de préférence pour le futur proche (plutôt qu'un futur éloigné) qui est d'autant plus élevé que le participant a choisi un grand nombre de fois le gain dans une semaine. Ce score correspond à un intervalle de taux d'escompte. Pour ce calcul, nous ne retenons que les individus cohérents dans leurs

\footnotetext{
2 - Une mignonette de chocolat correspond à un chocolat accompagnant un café. Sa valeur d'achat est de 0.15 euros.
} 
décisions, c'est-à-dire qui choisissent toujours le gain le plus proche ou toujours le gain le plus éloigné, ou qui ne changent qu'une fois du gain le plus proche vers le plus éloigné dans l'ordre chronologique des décisions. Pour l'ensemble des 394 participants cohérents avec les deux récompenses, les scores moyens (sur 10) de préférence pour le futur proche sont de 4,68 pour l'argent et de 4,83 pour le chocolat (un score de 10 indique que le participant préfère toujours le gain proche). La différence entre ces deux moyennes n'est pas significative (Wilcoxon matched-pairs signed-rank test : $\mathrm{Z}=-1,639 ; \mathrm{p}=0,101$ ).

Nous utilisons l'heure à laquelle les réponses ont été soumises en ligne afin d'analyser la variation, au cours de la journée, des préférences pour le futur proche avec les deux récompenses. Comme nous n'avons pas d'information relative à l'état de satiété du répondant, la possibilité qu'il y ait un lien entre satiété et préférences temporelles n'est ici qu'une extrapolation de la faim et de la satiété au cours de la journée, en fonction des repas. Par ailleurs, afin de tenir compte des pics de prises alimentaires (notamment midi et demi qui rassemble généralement $50 \%$ de la population), nous avons choisi un découpage de la journée allant d'une heure et demie à l'heure et demi suivante. Les scores moyens de préférence pour le futur proche associés au chocolat et à l'argent au cours de la journée sont représentés dans la figure $1^{3}$.

Figure 1. Score moyen de préférence pour le futur proche avec de l'argent (pointillés) et du chocolat (ligne pleine) en fonction des heures de la journée (en abscisse, le créneau horaire est suivi, entre crochets, du nombre d'observation) [ $\mathrm{N}=394]$

\footnotetext{
3 - Nous avons fait le choix de conserver l'ensemble des observations, même s'il y en a peu dans certains créneaux horaires. Une analyse excluant les créneaux ayant moins de 10 observations ne modifie pas qualitativement les résultats.
} 


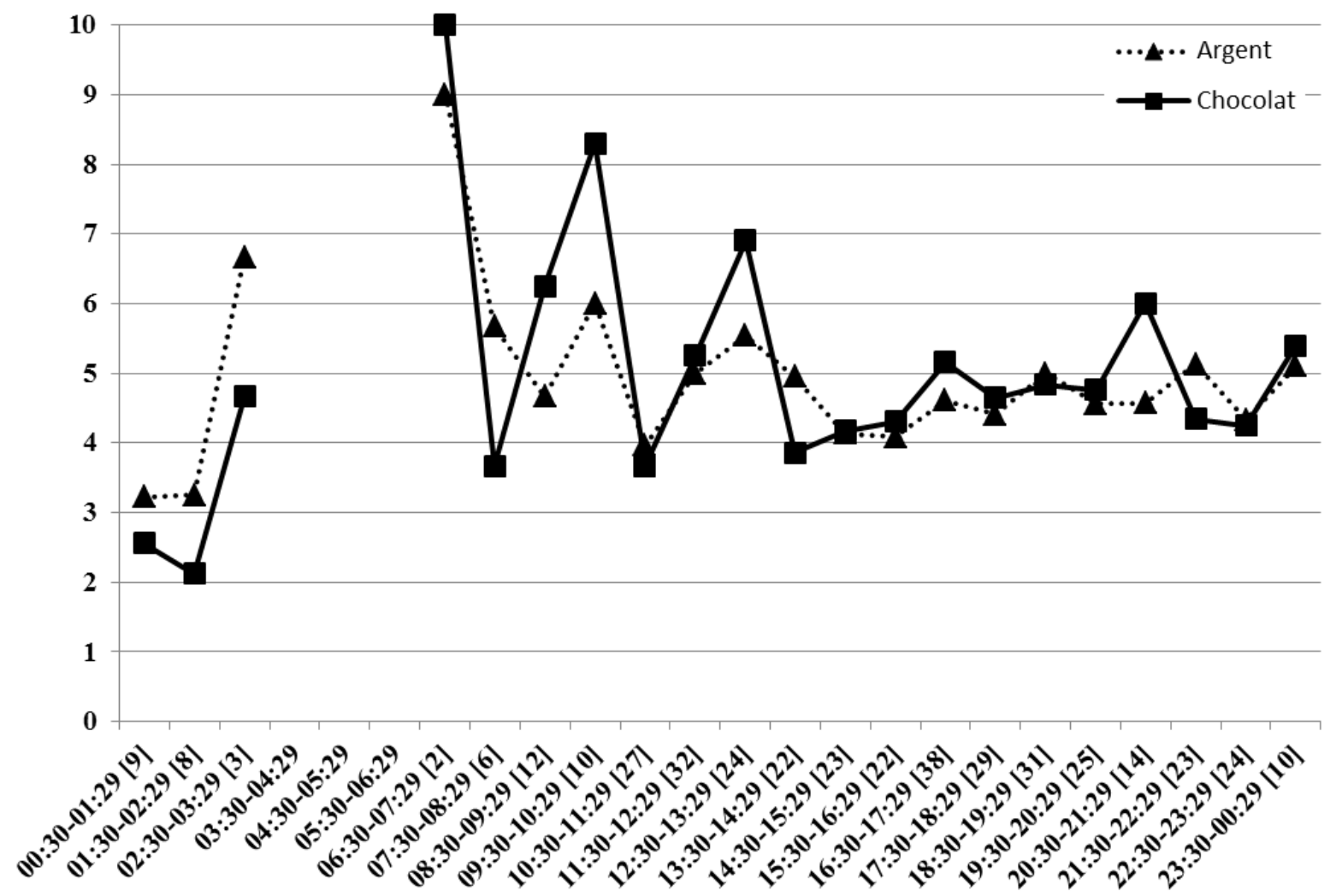

Un même individu connait un score pour chaque récompense (argent et chocolat). Les tests de comparaison intra-individuels par créneau horaires (Wilcoxon matched-pairs signedrank test) ne montrent pas de différences significatives entre les deux types de récompenses (excepté pour 4 créneaux sur 21 : [00:30-01:29], z=1,982; [01:30-02:29], z=1,72; [09:3010:29], $z=-1,806$; [20:30-21:29], z=-2,104). Ainsi, au sein d'un même créneau horaire, les deux mesures sont relativement homogènes et les individus plutôt cohérents.

Nous conduisons ensuite des comparaisons inter-individuelles à travers une analyse de la dynamique des arbitrages temporels pour l'argent et le chocolat au cours de la journée. Afin d'identifier des ruptures dans la journée, nous testons un à un les scores de chaque créneau horaire avec le précédent (Wilcoxon rank-sum test). Si aucune différence significative n'apparait pour les choix intertemporels liés à l'argent, certaines ruptures apparaissent pour ceux associés au chocolat. Ainsi, pour celui-ci, les différences significatives se situent entre la fin de matinée et le début d'après-midi. La préférence pour le futur proche diminue entre [09:30-10:29] et [10:30-11:29], puis augmente entre [10:30-11:29] et [11:30-12:29], augmente encore entre [11:30-12:29] et [12:30-13:29], puis diminue entre [12:30-13:29] et [13:30-14:29]. Le pic de préférence pour le futur proche associé au créneau horaire du déjeuner est ainsi particulièrement saillant. 
Une analyse paramétrique permet de tester cette observation par une estimation (régression linéaire) du score de préférence pour le futur proche en fonction des créneaux horaires et de caractéristiques individuelles (sexe, âge, année de l'enquête, goût pour le chocolat, score de préférence pour le futur proche avec l'autre bien afin de tenir compte d'hétérogénéités potentielles). Les résultats sont détaillés dans le tableau 2. Le créneau horaire de référence est celui présentant les scores de préférence pour le futur proche les plus faibles et un nombre d'observations relativement important ([10:30-11:29], $\mathrm{n}=27$ ).

Tableau 2. Estimation (régression linéaire) du score de préférence pour le futur proche avec l'argent et avec le chocolat en fonction des créneaux horaires de la journée [ $\mathrm{N}=394]$

\begin{tabular}{|l|r|r|r|r|r|c|}
\hline & \multicolumn{2}{|c|}{ Argent } & \multicolumn{3}{|c|}{ Chocolat } \\
\hline Variable & Coef. & & E.T. & Coef. & & E.T. \\
\hline Femme & $-0,518$ & & $(0,346)$ & 0,282 & & $(0,303)$ \\
\hline Age & $-0,036$ & & $(0,167)$ & $-0,056$ & & $(0,146)$ \\
\hline Appréciation chocolat & $-0,057$ & & $(0,098)$ & 0,019 & & $(0,086)$ \\
\hline Préférence futur proche : chocolat & 0,753 & $* * *$ & $(0,044)$ & & & \\
\hline Préférence futur proche : argent & & & & 0,577 & $* * *$ & $(0,034)$ \\
\hline Créneaux horaires : & & & & & & \\
\hline $00: 30-01: 29$ & 0,029 & & $(1,230)$ & $-0,690$ & & $(1,076)$ \\
\hline $01: 30-02: 29$ & 0,573 & & $(1,291)$ & $-1,262$ & & $(1,129)$ \\
\hline $02: 30-03: 29$ & 1,965 & & $(1,940)$ & 0,591 & & $(1,701)$ \\
\hline $06: 30-07: 29$ & 0,070 & & $(2,360)$ & 3,420 & $*$ & $(2,058)$ \\
\hline $07: 30-08: 29$ & 1,346 & & $(1,463)$ & $-0,927$ & & $(1,282)$ \\
\hline $08: 30-09: 29$ & $-1,336$ & & $(1,135)$ & 2,151 & $* *$ & $(0,989)$ \\
\hline $09: 30-10: 29$ & $-1,392$ & & $(1,198)$ & 3,451 & $* * *$ & $(1,035)$ \\
\hline $10: 30-11: 29$ (référence) & & & & & & \\
\hline $11: 30-12: 29$ & $-0,146$ & & $(0,843)$ & 1,003 & & $(0,736)$ \\
\hline $12: 30-13: 29$ & $-0,896$ & & $(0,911)$ & 2,390 & $* * *$ & $(0,789)$ \\
\hline $13: 30-14: 29$ & 0,884 & & $(0,922)$ & 0,410 & & $(0,808)$ \\
\hline $14: 30-15: 29$ & $-0,197$ & & $(0,904)$ & 0,409 & & $(0,791)$ \\
\hline $15: 30-16: 29$ & $-0,565$ & & $(0,930)$ & 0,629 & & $(0,814)$ \\
\hline $16: 30-17: 29$ & $-0,595$ & & $(0,818)$ & 1,127 & & $(0,714)$ \\
\hline $17: 30-18: 29$ & $-0,339$ & & $(0,866)$ & 0,666 & & $(0,758)$ \\
\hline $18: 30-19: 29$ & $-0,089$ & & $(0,868)$ & 0,580 & & $(0,759)$ \\
\hline $19: 30-20: 29$ & $-0,224$ & & $(0,888)$ & 0,706 & & $(0,777)$ \\
\hline $20: 30-21: 29$ & $-1,272$ & & $(1,059)$ & 1,992 & $* *$ & $(0,923)$ \\
\hline $21: 30-22: 29$ & 0,601 & & $(0,906)$ & $-0,012$ & & $(0,794)$ \\
\hline $22: 30-23: 29$ & $-0,023$ & & $(0,895)$ & 0,342 & & $(0,783)$ \\
\hline $23: 30-00: 29$ & $-0,249$ & & $(1,182)$ & 1,077 & & $(1,034)$ \\
\hline Année : 2016 (réf. $: 2015)$ & 0,305 & & $(0,368)$ & $-0,006$ & & $(0,338)$ \\
\hline Constante & 2,428 & & $(3,632)$ & 2,299 & & $(3,180)$ \\
\hline Nombre d'observations & & 394 & & & & 394 \\
\hline F(25; 368) & & $12,28 * * *$ & & $14,18 * * *$ \\
\hline R2 & & 0,454 & & & 0,491 \\
\hline
\end{tabular}


Note : les créneaux [03:30-04:29], [04:30-05:29] et [05:30-06:29] ne sont pas observés.

Coef. : coefficient ; E.T. : écart-type ; réf. : référence

Seuils de significativité : $*=10 \%, * *=5 \%, * * *=1 \%$

Le premier résultat est que les choix intertemporels pour les deux biens sont significativement et positivement corrélées (ce résultat est cohérent avec celui de Reuben et al. [2010]). Par ailleurs, quelle que soit l'estimation (argent et chocolat), les caractéristiques individuelles (âge, sexe) ne sont pas significatives. Le niveau d'appréciation du chocolat n'a pas d'effet non plus. Enfin, l'année de l'enquête n'a pas d'effet. L'échantillon est ainsi relativement homogène.

Le deuxième résultat, plus important, concerne la relation entre les choix intertemporels et l'heure à laquelle la décision est prise dans la journée. Si le moment de la décision dans la journée n'a aucun effet significatif en ce qui concerne l'argent, il n'en va pas de même pour le chocolat. Par rapport au créneau de référence, cinq créneaux horaires rassemblés en trois espaces temps de la journée apparaissent comme ayant un effet significatif et positif sur cette préférence (augmentation de l'impatience) : (1) la matinée ([06:30-07:29], [08:30-09:29] et [09:30-10:29]), (2) le midi ([12:30-13:29]), et (3) en soirée ([20:30-21:29]). Ces trois espaces de temps correspondent aux prises habituelles des repas en France, avec comme hypothèse que ces prises sont associées à la faim.

Nous pouvons modérer ces résultats sans pour autant rejeter les pistes de réflexions qu'ils ouvrent. Outre le fait que le premier créneau significatif de matinée implique peu d'observations ([06:30-07:29], $n=2$ ), la question se pose de savoir pourquoi les créneaux de [08:30-09:29] et de [09:30-10:29] présentent une plus forte préférence pour le futur proche avec le chocolat, alors qu'ils arrivent potentiellement après le petit-déjeuner. Une explication est que ce repas n'est pas pris par 4 jeunes de moins de 25 ans sur 10, contre 1 sur 10 pour les plus de 50 ans (De Saint Pol et Ricroch [2012]). Cette faim pourrait être rassasiée au court d'une pause-café qui correspond à la période de référence ([10:30-11:29]), période qui présente de faibles taux de préférence pour le futur proche (mais cela reste une conjecture).

Ainsi, des moments de la journée associés à la faim et à la prise alimentaire augmentent le taux de préférence pour le futur proche avec du chocolat (une denrée à même de recouvrir rapidement la faim), mais n'ont pas d'effets relatifs au même indicateur avec de l'argent. Dans ce cadre, l'hypothèse 1 ne serait pas rejetée pour le chocolat, mais le serait pour l'argent. Cela correspond aux résultats de Skrynka et Vincent [2017] et de Orquin et Kurzban [2016]. Par ailleurs, contrairement aux autres expériences, il ne s'agit pas d'une préférence pour l'immédiateté dans la mesure où le gain le plus proche est dans une semaine. Cependant, aux 
vues des résultats, les individus semblent se comporter comme si ce moment futur était comparable à celui de la prise de décision. Il pourrait ainsi y avoir un effet de projection non biaisé (i.e., de l'empathie temporelle). Les questions de cohérence dynamique ne peuvent cependant pas être abordées dans ce contexte expérimental.

\section{CONCLUSION}

Plusieurs expériences conduites dans différents environnements montrent un lien significatif entre l'état physiologique associé à la faim et les arbitrages intertemporels des individus (notamment lorsqu'il s'agit d'aliments). Cette relation n'est pas anodine. En effet, l'orientation de ces arbitrages, et a fortiori la préférence pour le présent, est associée à l'autocontrôle et à l'impulsivité alimentaire qui est elle-même corrélée à l'obésité (Benard et al. [2017]). Par ailleurs, Lohéac et al. [2012] observent que les individus ayant une préférence pour le présent ne valorisent pas des informations nutritionnelles. Enfin, Sweeney et Culcea [2017] observent dans leur méta-analyse une relation faible, mais significative, entre l'orientation vers le futur et les comportements sains en termes de santé. De plus, elles observent qu'il est possible de modifier cette caractéristique. Ainsi, la maîtrise ou l'anticipation de la faim peuvent constituer des paramètres agissant sur les arbitrages intertemporels, sur les décisions alimentaires, et par conséquent sur la santé des consommateurs. Ces éléments présentent un intérêt en termes de proposition de dispositifs de régulation comportementale. Ainsi, dans leurs expériences, VanEpps et al. [2016] montrent qu'en faisant commander à des gens leur déjeuner bien avant l'heure de celui-ci (c'est-à-dire plutôt en état de satiété), ils réduisent significativement les calories ingérées ${ }^{4}$. Ces expériences sur les relations des comportements avec l'état de satiété sont relativement récentes mais présentent des résultats encourageants en termes d'interventions comportementales. Des approfondissements sont à conduire sur les arbitrages et les incitations liées, notamment aux qualités des aliments, ainsi qu'à la régularité des comportements.

\section{REFERENCES}

\footnotetext{
4 - En dehors de considérations intertemporelles, mais en mobilisant le self-control et l'impulsivité, Cheung et al. [2017] observent que la faim réduit la préférence pour des aliments sains par rapport à la satiété. Cependant, ils montrent que cette préférence peut être modifiée par la présentation de statistiques mettant en avant le choix d'aliment sains par d'autres personnes (préférences sociales).
} 
ASHTON L. [2015], «Hunger games: Does hunger affect time preferences?», Working Paper, Disponible sur SSRN: https://ssrn.com/abstract=2538740.

BELLISLE F. [2005], «Faim et satiété, contrôle de la prise alimentaire », EMCEndocrinologie, 2 (4), p. 179-197.

BENARD M., CAMilleRI G.M., ETILE F., MEJEAN C., BELliSle F., REACH G., HERCBERG S. et PENEAU S. [2017], «Association between impulsivity and weight status in a general population », Nutrients, 9 (3), p. 217.

BERNHEIM B.D. et RANGEL A. [2004], «Addiction and cue-triggered decision processes », American Economic Review, 94 (5), p. 1558-1590.

BRIZ T., DRICHOUTIS A. et HOUSE L. [2015], «Examining projection bias in experimental auctions: the role of hunger and immediate gratification », Agricultural and Food Economics, 3 (22), p. 1-17.

CHEUNG T.T.L., KROESE F.M., FENNIS B.M. et De RIDDER D.T.D. [2017], «The hunger games: Using hunger to promote healthy choices in self-control conflicts », Appetite, 116, p. 401-409.

CORNIL Y. [2017], «Mind over stomach: A review of the cognitive drivers of food satiation », Journal of the Association for Consumer Research, 2 (4), p. 419-429.

DE MAGISTRIS T. et GRACIA A. [2016], «Assessing projection bias in consumers' food preferences », PLoS ONE Public Library of Science, 11 (2), p. 1-11.

De SAINT POL T. et RICROCH L. [2012], «Le temps de l'alimentation en France », INSEE Première, $\mathrm{n}^{\circ} 1417$.

FREDERICK S., LOEWENSTEIN G. et O'DONOGHUE T. [2002], « Time discounting and time preference: A critical review », Journal of Economic Literature, 40 (2), p. 351-401.

GILBERT D.T., GILL M.J. et WILSON T.D. [2002], «The future is now: Temporal correction in affective forecasting », Organizational Behavior and Human Decision Processes, 88 (1), p. 430-444.

HALEVY Y. [2015], « Time consistency: Stationarity and time invariance », Econometrica, 83 (1), p. 335-352.

HEISLER L.K. et LAM D.D. [2017], «An appetite for life: brain regulation of hunger and satiety », Current Opinion in Pharmacology, 37, p. 100-106.

KUHN M.A., KUHN P. et VILLEVAL M.C. [2017], «Decision-environment effects on intertemporal financial choices: how relevant are resource-depletion models? », Journal of Economic Behavior \& Organization, 137, p. 72-89. 
LAIBSON D.I. [1997], «Golden eggs and hyperbolic discounting », Quarterly Journal of Economics, 112 (2), p. 443-478.

LAIBSON D.I. [2001], «A cue-theory of consumption », Quarterly Journal of Economics, $116(1)$, p. 81-119.

LHUISSIER A., TICHIT C., CAILlAVET F., CARDON P., MASUllO A., MARTINFERNANDEZ J., PARIZOT I. et CHAUVIN P. [2013], « Who still eats three meals a day? Findings from a quantitative survey in the Paris area », Appetite, 63, p. 59-69.

LOHEAC Y., COMBRIS P. et ISSANCHOU S. [2012], « Information nutritionnelle, choix et caractéristiques des consommateurs », Revue Française d'Economie, 26 (3), p. 29-68.

LOEWENSTEIN G. [1996], «Out of control: Visceral influences on behavior», Organizational Behavior and Human Decision Processes, 65 (3), p. 272-92.

LOEWENSTEIN G. [2000], «Emotions in economic theory and economic behavior», American Economic Review, 90 (2), p. 426-432.

LOEWENSTEIN G. [2005], « Hot-cold empathy gaps and medical decision making », Health Psychology, 24 (4S), p. S49-S56.

LOEWENSTEIN G., O'DONOGHUE T. et RABIN M. [2003], «Projection biais in predicting future utility », Quarterly Journal of Economics, 118 (4), p. 1209-1248.

NIWANO Y., ADACHI T., KASHIMURA J., SAKATA T., SASAKI H., SEKINE K., YAMAMOTO S., YONEKUBO A. et KIMURA S. [2009], «Is glycemic index of food a feasible predictor of appetite, hunger, and satiety?», Journal of Nutritional Science and Vitaminology, 55 (3), p. 201-207.

ORQUIN J.L. et KURZBAN R. [2016], « A meta-analysis of blood glucose effects on human decision making », Psychological Bulletin, 142 (5), p. 546-567.

PENICAUD L. et FIORAMONTI J. [2015], «Bases physiologiques de la satiété et de la digestion », dans C. ESNOUF, J. FIORAMONTI et B. LAURIOUX (dir.), L'Alimentation à découvert, CNRS Editions, p. 23-24.

READ D. et VAN LEEUWEN B. [1998], «Predicting hunger: The effects of appetite and delay on choice », Organizational Behavior and Human Decision Process, 76 (2), p. 189205.

REUBEN E., SAPIENZA P. et ZINGALES L. [2010], « Time discounting for primary and monetary rewards », Economic Letters, 106 (2), p. 125-127.

SCHWARTZ M.B., JUST D.R., CHRIQUI J.F. et AMMERMAN A.S. [2017], « Appetite self-regulation: Environmental and policy influences on eating behaviors », Obesity, 25 (S1), p. S26-S38. 
SKRYNKA J. et VINCENT B.T. [2017], « Hunger Increases Delay Discounting of Food and Non-food Rewards », Working Paper, PsyArXiv, Open Science Framework, http://doi.org/10.17605/OSF.IO/QGP54.

SWEENEY A.M. et CULCEA I. [2017], « Does a future-oriented temporal perspective relate to body mass index, eating, and exercise? A meta-analysis », Appetite, 112, p. 272-285.

VANEPPS E.M., DOWNS J.S., LOEWENSTEIN G. [2016], «Advance ordering for healthier eating? Field experiments on the relationship between the meal orderconsumption time delay and meal content », Journal of Marketing Research, 53 (3), p. 369-380.

WANG X.T. et HUANGFU G. [2017], «Glucose-specific signaling effects on delay discounting in intertemporal choice », Physiology \& Behavior, 169, p. 195-201. 